\title{
Role of Peritoneal Lavage Cytology and Prediction of Prognosis and Peritoneal Recurrence After Curative Surgery for Colorectal Cancer
}

\author{
Sung Joon Bae*, Ui Sup Shin*, Young-Jun Ki, Sang Sik Cho, Sun Mi Moon, Sun Hoo Park ${ }^{1}$ \\ Departments of Surgery and ${ }^{1}$ Pathology, Korea Cancer Center Hospital, Korea Institute of Radiological \& Medical Sciences, Seoul, Korea
}

Purpose: In colorectal cancer, the role of detecting free malignant cells from peritoneal lavage is currently unclear. In this study, we investigated the positive rate of free malignant cells in peritoneal lavage fluid and their predictive value for prognosis and peritoneal recurrence after a curative resection.

Methods: From October 2009 to December 2011, in a prospective manner, we performed cytologic examinations of peritoneal lavage fluid obtained just after the abdominal incision from 145 patients who underwent curative surgery for colorectal cancer. We used proportional hazard regression models to analyze the predictive role of positive cytology for peritoneal recurrence and survival.

Results: Among total 145 patients, six patients (4.1\%) showed positive cytology. During the median follow-up of 32 months (range, 8-49 months), 27 patients (18.6\%) developed recurrence. Among them, 5 patients (3.4\%) showed peritoneal carcinomatosis. In the multivariate analysis, positive cytology was an independent predictive factor for peritoneal recurrence (hazard ratio [HR], 136.5; 95\% confidence interval [CI], 12.2-1,531.9; $\mathrm{P}<0.0001$ ) and an independent poor prognostic factor for overall survival (HR, 11.4; 95\% CI, 1.8-72.0; P = 0.009) and for disease-free survival (HR, 11.1; 95\% CI, 3.4-35.8; $\mathrm{P}<0.0001)$.

Conclusion: Positive cytology of peritoneal fluid was significantly associated with peritoneal recurrence and worse survival in patients undergoing curative surgery for colorectal cancer. Peritoneal cytology might be a useful tool for selecting patients who need intraperitoneal or systemic chemotherapy.

Keywords: Peritoneal metastasis; Cytology; Colorectal neoplasms; Survival; Prognosis

\section{INTRODUCTION}

During the last two decades, the prognosis for patients with metastatic colorectal cancer (mCRC) has been noticeably improved because of the current combinations of chemotherapies with target agents and the development of surgical techniques. Surgical resec-

Received: July 21, 2014 - Accepted: October 14, 2014

Correspondence to: Ui Sup Shin, M.D.

Department of Surgery, Korea Cancer Center Hospital, Korea Institute of Radiological \& Medical Sciences, 75 Nowon-ro, Nowon-gu, Seoul 139-706, Korea

Tel: +82-2-970-1216, Fax: +82-2-970-2419

E-mail: uisup.shin@gmail.com

*Sung Joon Bae and Ui Sup Shin contributed equally as co-first authors.

(C) 2014 The Korean Society of Coloproctology

This is an open-access article distributed under the terms of the Creative Commons Attribution NonCommercial License (http://creativecommons.org/licenses/by-nc/3.0) which permits unrestricted noncommercial use, distribution, and reproduction in any medium, provided the original work is properly cited. tion of hepatic and pulmonary metastatic lesions can achieve longterm survival of more than three years $[1,2]$. Moreover, the survival of patients with unresectable mCRCs has been improved, up to 30 months, in the era of contemporary systemic chemotherapy with biologic agents [3]. However, these improvements in survival are not consistent among all mCRC patients. In mCRC with peritoneal carcinomatosis (PC), progress in multidisciplinary treatment has resulted in only a limited survival benefit $[4,5]$.

Peritoneal cytology has been considered to be useful for predicting an individual prognosis for some malignancies. Keettel and Elkin [6] introduced the technique of intraoperative peritoneal washing cytology in ovarian cancer patients for the first time in 1956. In 1975, the International Federation of Gynecologists \& Obstetricians incorporated results of peritoneal cytology into the staging classification for ovarian cancer, and in 1989. It did so for endometrial cancer. Among the nongynecological adenocarcinomas, especially gastric and pancreatic adenocarcinomas, the pres- 
ence of free malignant cells in the peritoneal fluid is associated with poor survival and peritoneal recurrence and in 2009 was integrated as part of the American Joint Committee on Cancer (AJCC) TNM classification for cancers like uterine and ovarian cancers 2009 [7]. In colorectal cancer, there have been many reports on a positive correlation with poor prognosis and prediction of peritoneal recurrence $[8,9]$. However, those results are still being debated [10]. New treatment modalities, including aggressive cytoreductive surgery with hyperemic intraperitoneal chemotherapy (HIPEC) and early intraperitoneal chemotherapy (EPIC) have been developed to obtain long-term control of $\mathrm{PC}$ and to maintain long-term PC-free survival $[11,12]$. Knowing that the target for HIPEC or EPIC is intraperitoneal free cancer cells or tiny nodules, not gross disease, positive peritoneal cytology could represent an adequate selection factor following such aggressive treatments. Accordingly, revealing the correlation of intraperitoneal free cancer cells to survival and peritoneal recurrence is of primary importance and would lead to uniform therapeutic decision-making and meticulous postoperative follow-up. In this study, we investigated the tumor-positive rate of peritoneal washing cytology in patients with CRC who underwent curative surgery, and we analyzed the association of positive cytology with prognosis and peritoneal recurrence.

\section{METHODS}

\section{Patients}

From October 2009 to December 2011, we prospectively collected data on 145 patients who underwent a curative resection for colorectal cancer. Enrolled patients had no evidence of distant metastasis at preoperative staging work-up, including abdomino-pel- vic computed tomography (CT), chest CT and 18-fluorodeoxyglucose positron emission tomography scan. Patients who had a previous history of treatment for malignancy, Lynch syndrome, or familial adenomatous polyposis were excluded from this study. Patients with unrecognized PC or hepatic involvement at surgical exploration were also excluded. The pathologic staging of cancer was assessed postoperatively according to the seventh edition of the AJCC TNM grading system [7]. Charlson's comorbidity index was calculated to evaluate the status of patients' comorbidity. The Institutional Review Board of Korea Cancer Center Hospital approved this study, and written informed consent for tissue collection was obtained from all patients.

\section{Procedures}

Peritoneal lavage was performed immediately after we had made a midline abdominal incision and just before we manipulated the tumor. About $100 \mathrm{~mL}$ of physiologic saline solution $\left(37^{\circ} \mathrm{C}\right)$ was instilled into the abdominal cavity around the tumor with the patient in a supine position. After gentle stirring, these fluids were collected with a suction device at the Douglas pouch.

All peritoneal lavage specimens were prepared using the ThinPrep liquid-based cytology preparation system (Cytyc Co., Boxborough, MA, USA). The sample was centrifuged at $600 \mathrm{~g}$ for 10 minutes, and the supernatant was poured off carefully. The cell pellet was resuspended and washed with $30 \mathrm{~mL}$ of CytoLyt solution. The specimen was added to a PreservCyt (Cytyc Co.) solution vial and allowed to stand for 15 minutes. The vial was then placed in a Cytyc ThinPrep 2000 processor utilizing a computerized process and patented membrane technology for dispersion control, collection, and transfer of diagnostic cells from the sample to a $20-\mathrm{mm}$ circular area on a glass slide. The slide was fixed
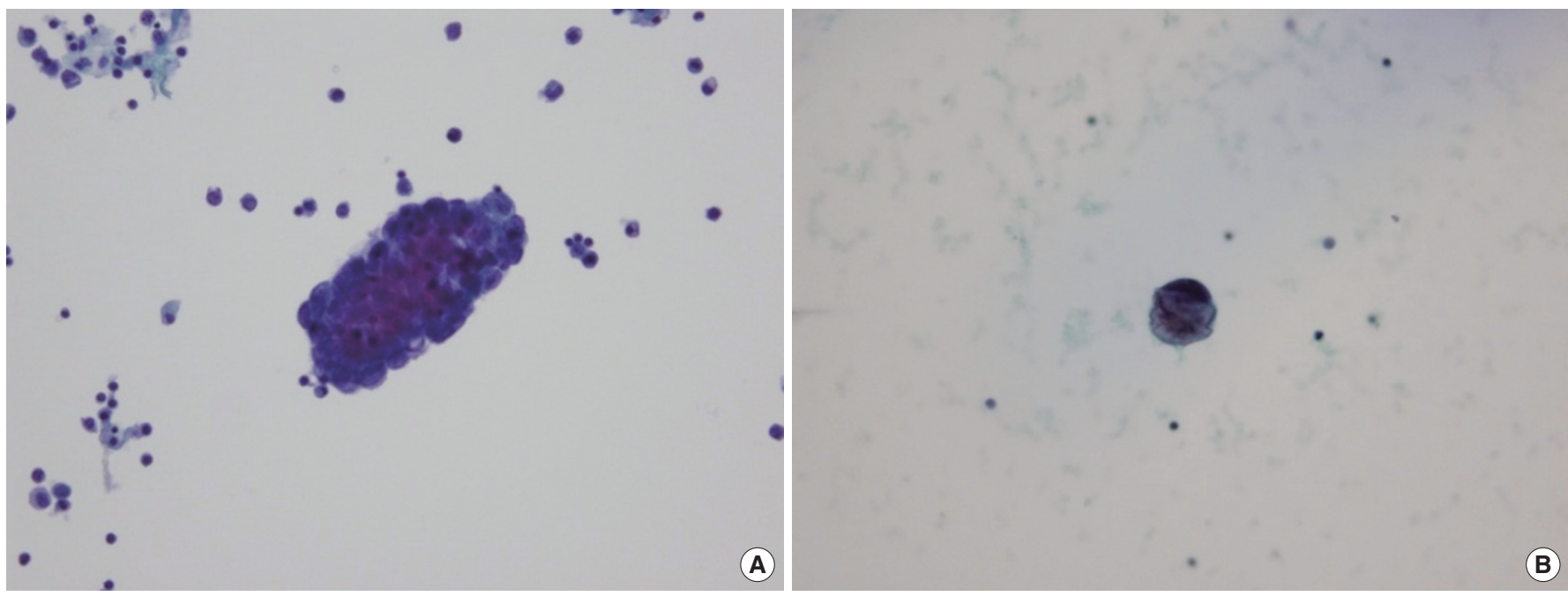

Fig. 1. (A) The malignant cells are arranged in 3-dimensional clusters with overlapping nuclei in the background of scattered reactive mesothelial cells, neutrophils and lymphocytes (Papanicolaou staining, $\times 400$ ). (B) A tumor cell with high nuclear-to-cytoplasmic ratio has a hyperchromatic and coarse nucleus with an irregular eccentric contour and is 10 times larger than lymphocytes (Papanicolaou staining, $\times 400)$. 
in 95\% ethanol and stained by using the Papanicolaou and the diastase-periodic acid-Schiff staining methods.

All ThinPrep slides were reviewed and diagnosed by an experienced pathologist with a specialization in gastrointestinal oncology. A slide was classified as positive if malignant cells formed three-dimensional clusters or one malignant cell had a high nuclear cell ratio and over 10-fold enlargement compared to an adjacent lymphocyte (Fig. 1). A suspicion of malignancy or atypical finding was classified as negative.

\section{Follow-up}

The median duration of the follow-up period for all patients was 32 months (8-49 months). For detection of recurrence during the follow-up period, physical examinations and CEA checks were done every 3 months, and CT scans were done every 6 months in first two years after operation. Since then, carcinoembryonic antigen (CEA) checks and CT scans, including physical examinations, have been done every 6 months. Peritoneal and any systemic recurrences were diagnosed based on radiologic and/or pathologic evidence of cancer recurrence.

\section{Statistical analysis}

Statistical analyses were performed using the SPSS ver. 14.0 (SPSS Inc., Chicago, IL, USA). Associations between the clinicopathologic parameters were assessed using the chi-square test or the Fisher exact test for categorical variables. For continuous variables, the independent sample t-test or Mann Whitney U-test were done appropriately. Disease-free survivals (DFSs) and overall survivals (OSs) were calculated by using the Kaplan-Meier methods and were compared by using the log-rank test. The Cox proportional hazard model with a backward elimination method was used for multivariate analyses. In univariate analyses, variables whose P-values were less than 0.05 were selected for the multivariate analysis. P-values of less than 0.05 were considered statistically significant.

\section{RESULTS}

Overall, 6 of the 145 patients (4.1\%) showed positive malignant cells in the peritoneal lavage fluid. All six positive cytology results were found in patients with more than T3 stage cancer. However, the distributions of pathologic $\mathrm{T}$ stages were not significantly different between the two groups according to the results of peritoneal cytology $(\mathrm{P}=0.74)$. The rate of tumor perforation was relatively higher in patients with positive cytology than in patients with negative cytology, but the difference was not statistically significant. Mucinous phenotype or poor histologic grade was more frequently found in the positive-cytology group with statistical significance $(\mathrm{P}=0.008)$ (Table 1$)$.

During the follow-up period, 27 patients showed recurrence. Among them, 5 patients (18.5\%, 3.4\% of total) developed peritoneal recurrence. Four of these 5 patients were in the positive-cytology group. Other sites of recurrence were not significantly re- lated with positive cytology (Table 2).

The 3-year DFS rate and OS rate for all patients were $77.0 \%$ and $93.2 \%$, respectively. Comparing the DFS according to status of the peritoneal cytologic results, the 3-year DFS of the patients with positive cytology was $30.0 \%$, and that of the patients with negative cytology was $78.5 \%(\mathrm{P}=0.001)$ (Fig. 2). Also, the 3 -year OS of the patients with positive cytology was $66.7 \%$, which was significantly

Table 1. Patients' characteristics and factors associated with positive cytology

\begin{tabular}{|c|c|c|c|c|}
\hline & $\begin{array}{l}\text { Cytology }(+) \\
(n=6)\end{array}$ & $\begin{array}{c}\text { Cytology (-) } \\
(\mathrm{n}=139)\end{array}$ & $\begin{array}{c}\text { Total } \\
(\mathrm{n}=145)\end{array}$ & P-value \\
\hline Age (yr) & $60.8 \pm 9.7$ & $62.4 \pm 11.1$ & $62.4 \pm 11.1$ & 0.67 \\
\hline Male gender & $2(33.3)$ & 78 (56.1) & 80 (55.2) & 0.41 \\
\hline Charlson score & $5.5 \pm 1.5$ & $5.6 \pm 1.7$ & $5.6 \pm 1.7$ & 0.86 \\
\hline $\begin{array}{l}\text { Adjuvant } \\
\text { chemotherapy }\end{array}$ & $6(100)$ & $102(73.4)$ & 108 (74.5) & 0.34 \\
\hline 5-FU only & $1(16.7)$ & $66(47.5)$ & 67 (46.2) & 0.01 \\
\hline $\begin{array}{l}\text { 5-FU, } \\
\text { oxaliplatin }\end{array}$ & $5(83.3)$ & $36(25.9)$ & 41 (28.3) & \\
\hline None & $0(0)$ & 37 (26.6) & 37 (25.5) & \\
\hline Rectum & $1(16.7)$ & 69 (49.6) & 70 (48.3) & 0.21 \\
\hline \multicolumn{5}{|l|}{ T stage } \\
\hline pT0, T1, T2 & $0(0)$ & 45 (32.4) & 45 (31.0) & 0.17 \\
\hline pT3 & $5(83.3)$ & 77 (55.4) & $82(56.6)$ & \\
\hline pT4 & $1(16.7)$ & 17 (12.2) & $18(12.4)$ & \\
\hline Node metastasis & $2(33.3)$ & $33(23.7)$ & 35 (24.1) & 0.63 \\
\hline Stage 0 (CR) & $0(0)$ & $7(5.0)$ & & 0.62 \\
\hline I & $0(0)$ & $34(24.5)$ & & \\
\hline$\|$ & $4(66.7)$ & 65 (46.8) & & \\
\hline III & $2(33.3)$ & $33(23.7)$ & & \\
\hline $\begin{array}{l}\text { Lymphatic } \\
\text { invasion }\end{array}$ & $2(33.3)$ & 33 (23.7) & $35(24.1)$ & 0.63 \\
\hline Vascular invasion & $0(0)$ & $14(10.1)$ & $14(9.7)$ & 1.00 \\
\hline $\begin{array}{l}\text { Perineural } \\
\text { invasion }\end{array}$ & $2(33.3)$ & 24 (17.3) & $26(17.9)$ & 0.29 \\
\hline $\begin{array}{c}\text { Differentiation } \\
\text { (PD/MUC) }\end{array}$ & $3(50.0)$ & $9(6.5)$ & $12(8.3)$ & 0.008 \\
\hline Obstruction & $0(0)$ & 15 (10.9) & 15 (10.3) & 1.00 \\
\hline Perforation & $1(16.7)$ & $2(1.4)$ & $3(2.1)$ & 0.12 \\
\hline CEA (ng/mL) & $74.0 \pm 159.2$ & $8.2 \pm 17.4$ & $10.2 \pm 35.9$ & 0.36 \\
\hline $\begin{array}{l}\text { CA 19-9, } \\
\text { median (range) }\end{array}$ & $14.9(5.0-24.6)$ & $9.9(0.6-205.7)$ & $10.1(0.6-205.7)$ & 0.67 \\
\hline
\end{tabular}

Values are presented as mean \pm standard deviation or number (\%) unless otherwise indicated.

5-FU, 5-fluorouracil; CR, complete remission; PD, poorly differentiated adenocarcinoma; MUC, mucinous adenocarcinoma; CEA, carcinoembryonic antigen; CA 19-9, carbohydrate antigen 19-9. 
worse than that of the patients with negative cytology (94.4\%, P = 0.002) (Fig. 3). Regarding the peritoneal-recurrence-free rate, the patients with positive cytology showed significantly worse outcome than those with negative cytology $(25.0 \%$ vs. $99.2 \%, \mathrm{P}<$ 0.0001) (Fig. 4).

To find the factors impacting the DFS, OS and peritoneal-recurrence-free survival, we performed uni- and multivariate Cox regression analyses with clinicopathologic variables. In the univariate analysis, positive cytology was a significant poor prognostic factor that affected DFS (hazard ratio [HR], 7.15; 95\% confidence interval [CI], 2.44-20.96; P < 0.0001). Pathologic T4 stage, regional lymph-node metastasis, lymphatic, perineural, and vascular invasion, and tumor perforation were also significantly associated with the DFS in the univariate analysis. In the multivariate analysis, positive cytology was one of the independent factors affecting the DFS (HR, 11.05; 95\% CI, 3.41-35.8; P < 0.0001), along with regional lymph-node metastasis and the status of vascular and perineural invasion (Table 3).

In the analyses of the OS, a positive cytologic result was significantly associated with poor prognosis in the univariate analysis (HR, 8.49; 95\% CI, 1.7-42.51; P = 0.009) as revealed by a former analysis. Regional lymph-node metastasis, lymphatic, vascular

Table 2. Site of recurrence according to cytologic results

\begin{tabular}{lccrc}
\hline Site of recurrence & $\begin{array}{c}\text { Positive cytology } \\
(\mathrm{n}=6)\end{array}$ & $\begin{array}{c}\text { Negative cytology } \\
(\mathrm{n}=139)\end{array}$ & $\begin{array}{c}\text { Total } \\
(\mathrm{n}=145)\end{array}$ & P-value \\
\hline Peritoneum & $4(66.7)$ & $1(0.7)$ & $5(3.4)$ & $<0.0001$ \\
Liver & $1(16.7)$ & $7(5.0)$ & $8(5.5)$ & 0.29 \\
Lung & $0(0)$ & $12(8.6)$ & $12(8.3)$ & 1.00 \\
Distant lymph node & $1(16.7)$ & $4(2.9)$ & $5(3.4)$ & 0.19 \\
\hline
\end{tabular}

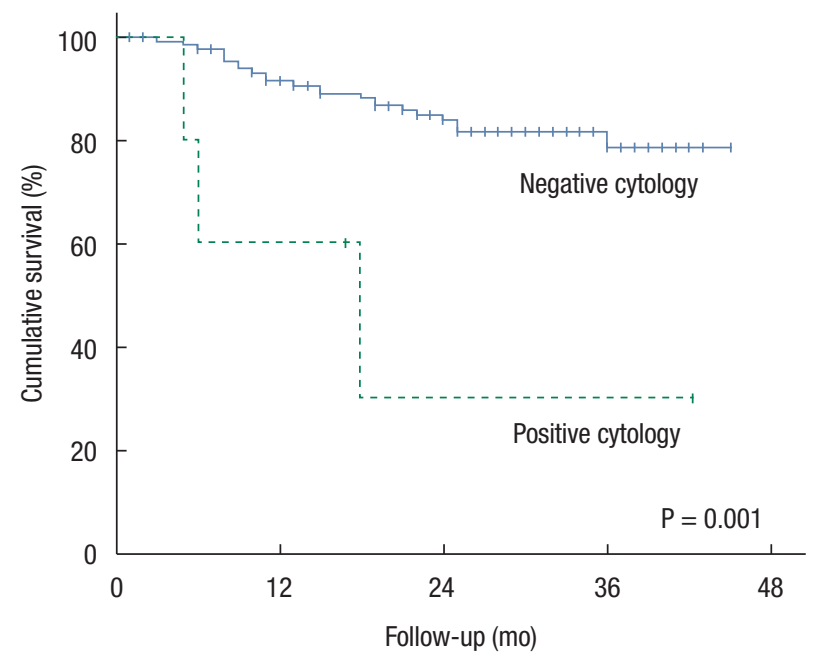

Fig. 2. Kaplan-Meier survival curves for disease-free survival according to the results of peritoneal cytology. and perineural invasion, and tumor perforation were additional poor prognostic factors. In the multivariate analysis, a positive cytologic result (HR, 11.43; 95\% CI, 1.82-71.95; P < 0.0001) was one of the independent prognostic factors for the OS, along with lymphatic and vascular invasion and tumor perforation (Table 4).

The Cox regression analyses for factors associated with peritoneal recurrence are presented in Table 5 . A positive cytologic result (HR, 136.5; 95\% CI, 12.17-1,531.91; $\mathrm{P}<0.0001$ ) and tumor perforation were the significant risk factors for peritoneal recurrence in the multivariate analysis. The pathologic T4 stage was significantly associated with peritoneal recurrence in the univariate analysis, but this significance disappeared in the multivariate analysis.

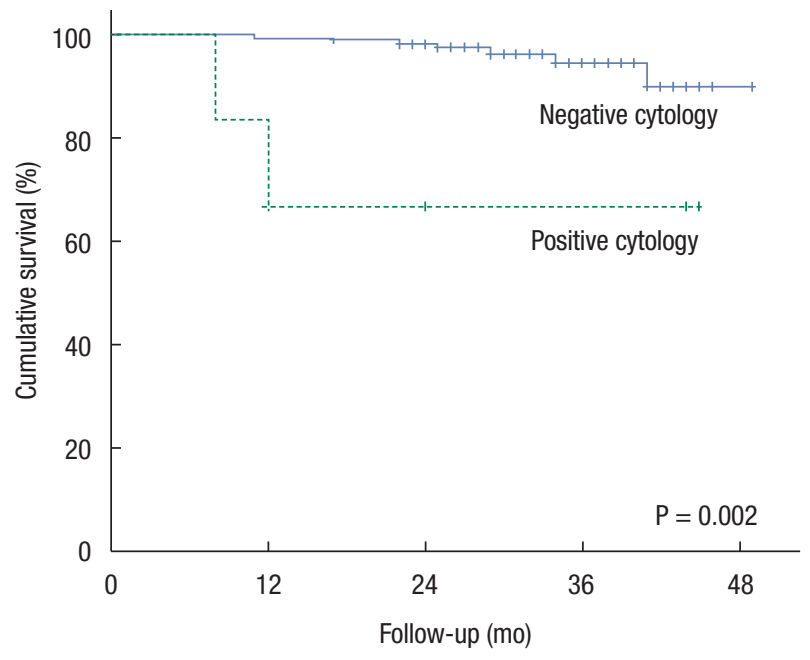

Fig. 3. Kaplan-Meier survival curves for overall survival according to the results of peritoneal cytology.

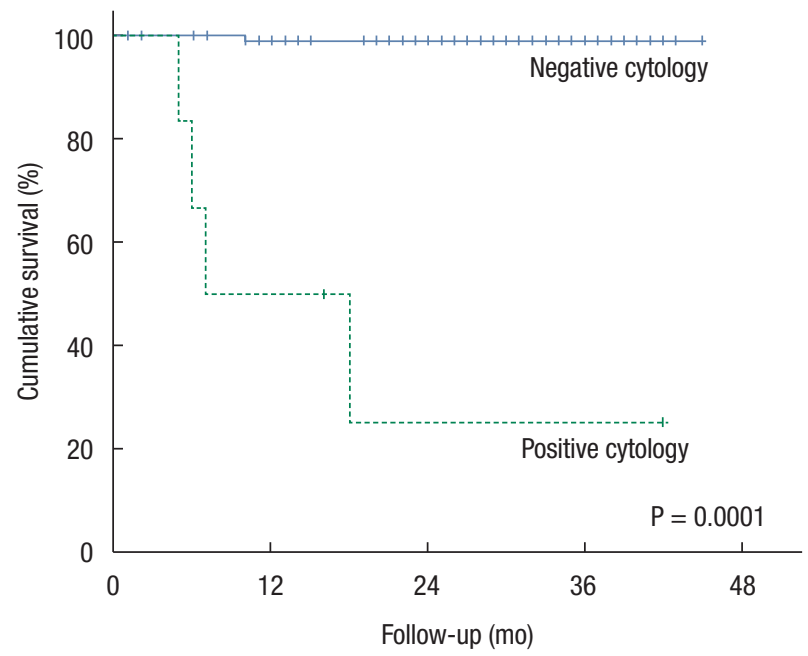

Fig. 4. Kaplan-Meier survival curves for peritoneal recurrence-free survival according to the results of peritoneal cytology. 
Table 3. Cox regression analyses for factors associated with disease-free survival

\begin{tabular}{|c|c|c|c|c|c|c|}
\hline \multirow{2}{*}{ Variable } & \multicolumn{3}{|c|}{ Univariate } & \multicolumn{3}{|c|}{ Multivariate } \\
\hline & HR & $95 \% \mathrm{Cl}$ & P-value & HR & $95 \% \mathrm{Cl}$ & P-value \\
\hline Male sex & 1.82 & $0.82-4.06$ & 0.142 & & & \\
\hline Charlson score $>6$ & 0.85 & $0.40-1.82$ & 0.676 & & & \\
\hline Adjuvant chemotherapy & 2.25 & $0.68-7.48$ & 0.185 & & & \\
\hline Rectum & 0.8 & $0.37-1.71$ & 0.563 & & & \\
\hline pT4 & 2.93 & $1.18-7.28$ & 0.021 & & & \\
\hline Node metastasis & 2.82 & $1.32-6.04$ & 0.007 & 2.90 & $1.33-6.33$ & 0.007 \\
\hline Lymphatic invasion & 3.25 & $1.52-6.96$ & 0.002 & & & \\
\hline Vascular invasion & 9.64 & $3.96-23.46$ & $<0.0001$ & 8.26 & $3.17-21.56$ & $<0.0001$ \\
\hline Perineural invasion & 5.64 & $2.62-12.17$ & $<0.0001$ & 3.49 & $1.53-7.94$ & 0.003 \\
\hline Differentiation (PD/MUC) & 1.89 & $0.57-6.29$ & 0.299 & & & \\
\hline Obstruction & 1.65 & $0.57-4.77$ & 0.358 & & & \\
\hline Perforation & 6.00 & $1.41-25.55$ & 0.015 & & & \\
\hline Positive cytology & 7.15 & 2.44-20.96 & $<0.0001$ & 11.05 & $3.41-35.80$ & $<0.0001$ \\
\hline $\mathrm{CEA}>7 \mathrm{ng} / \mathrm{mL}$ & 2.00 & $0.91-4.36$ & 0.83 & & & \\
\hline
\end{tabular}

$\mathrm{HR}$, hazard ratio; $\mathrm{Cl}$, confidence interval; PD, poorly differentiated adenocarcinoma; MUC, mucinous adenocarcinoma; CEA, carcinoembryonic antigen.

Table 4. Cox regression analyses for factors associated with overall survival

\begin{tabular}{|c|c|c|c|c|c|c|}
\hline \multirow{2}{*}{ Variable } & \multicolumn{3}{|c|}{ Univariate } & \multicolumn{3}{|c|}{ Multivariate } \\
\hline & HR & $95 \% \mathrm{Cl}$ & P-value & $\mathrm{HR}$ & $95 \% \mathrm{Cl}$ & P-value \\
\hline Male sex & 0.64 & $0.17-2.37$ & 0.501 & & & \\
\hline Charlson score $>6$ & 0.80 & $0.21-2.97$ & 0.733 & & & \\
\hline Adjuvant chemotherapy & 0.64 & $0.16-2.56$ & 0.527 & & & \\
\hline Rectum & 0.77 & $0.21-2.88$ & 0.699 & & & \\
\hline pT4 & 0.97 & $0.12-7.74$ & 0.974 & & & \\
\hline Node metastasis & 4.43 & $1.18-16.56$ & 0.027 & & & \\
\hline Lymphatic invasion & 5.91 & $1.47-23.72$ & 0.012 & 5.52 & $1.09-27.95$ & 0.039 \\
\hline Vascular invasion & 4.67 & $1.17-18.66$ & 0.029 & 5.43 & $0.99-29.95$ & 0.052 \\
\hline Perineural invasion & 6.08 & $1.63-22.63$ & 0.007 & & & \\
\hline Differentiation (PD/MUC) & 3.39 & $0.70-16.46$ & 0.130 & & & \\
\hline Obstruction & 1.38 & $0.17-11.18$ & 0.763 & & & \\
\hline Perforation & 30.23 & $5.95-153.55$ & $<0.0001$ & 82.42 & $10.35-656.58$ & $<0.0001$ \\
\hline Positive cytology & 8.49 & $1.70-42.51$ & 0.009 & 11.43 & $1.82-71.95$ & 0.009 \\
\hline $\mathrm{CEA}>7 \mathrm{ng} / \mathrm{mL}$ & 1.54 & $0.38-6.16$ & 0.540 & & & \\
\hline
\end{tabular}

$\mathrm{HR}$, hazard ratio; Cl, confidence interval; PD, poorly differentiated adenocarcinoma; MUC, mucinous adenocarcinoma; CEA, carcinoembryonic antigen.

\section{DISCUSSION}

In this study, the rate of a positive cytologic result for peritoneal lavage fluid in patients with colorectal cancer without distant metastasis was $4.1 \%$, and positive cytology was an independent poor prognostic factor for survival and a predictive factor for peritoneal recurrence. The rate of positive peritoneal cytology has been re- ported to range from $2.1 \%$ to $52 \%$ in patients with colorectal cancer [9]. This wide range across studies may be associated with heterogeneity of the techniques used to detect malignant cells in peritoneal lavage fluid. A recent systematic review for intraoperative peritoneal lavage reported mean weighted yields of $8.4 \%, 28.3 \%$, and $14.5 \%$ for conventional cytology, immunocytochemistry (ICC) and polymerase chain reaction (PCR), respectively [13]. The 
Table 5. Cox regression analyses for factors associated with peritoneal recurrence

\begin{tabular}{|c|c|c|c|c|c|c|}
\hline \multirow{2}{*}{ Variable } & \multicolumn{3}{|c|}{ Univariate } & \multicolumn{3}{|c|}{ Multivariate } \\
\hline & $\mathrm{HR}$ & $95 \% \mathrm{Cl}$ & P-value & HR & $95 \% \mathrm{Cl}$ & P-value \\
\hline Male sex & 1.24 & $0.21-7.40$ & 0.816 & & & \\
\hline Charlson score $>6$ & 1.44 & $0.24-8.65$ & 0.687 & & & \\
\hline Adjuvant chemotherapy & 0.34 & $0.004-271.16$ & 0.46 & & & \\
\hline pT4 & 5.65 & $0.94-33.86$ & 0.058 & & & \\
\hline Node metastasis & 4.65 & $0.78-27.84$ & 0.092 & & & \\
\hline Rectum & 0.26 & $0.03-2.33$ & 0.229 & & & \\
\hline Lymphatic invasion & 5.03 & $0.84-30.11$ & 0.077 & & & \\
\hline Vascular invasion & 0.04 & $0-9,640.64$ & 0.646 & & & \\
\hline Perineural invasion & 7.60 & $1.27-45.47$ & 0.026 & & & \\
\hline Differentiation (PD/MUC) & 21.50 & $3.57-129.53$ & 0.001 & & & \\
\hline Positive cytology & 142.54 & $15.57-1,305.22$ & $<0.0001$ & 136.52 & $12.17-1,531.91$ & $<0.0001$ \\
\hline Obstruction & 0.04 & $0-10,967.55$ & 0.618 & & & \\
\hline Perforation & 91.08 & $14.62-567.31$ & $<0.0001$ & 33.03 & $1.82-599.66$ & 0.018 \\
\hline $\mathrm{CEA}>7 \mathrm{ng} / \mathrm{mL}$ & 17.44 & $1.95-156.2$ & 0.01 & & & \\
\hline
\end{tabular}

$\mathrm{HR}$, hazard ratio; $\mathrm{Cl}$, confidence interval; PD, poorly differentiated adenocarcinoma; MUC, mucinous adenocarcinoma; CEA, carcinoembryonic antigen.

positive rates of ICC and PCR were relatively higher than the detection rate of conventional cytology. However, their significance as a prognostic factor for survival or a predictive factor for peritoneal recurrence is not clear [14]. ICC is subjective and depends on the strength of cellular staining, and PCR-based methods have inherent problems as they detect RNA, not viable cells, and cannot delineate cancerous cells from nonmalignant cells or cellular debris $[13,15]$. In addition, the targeted antigen or RNAs are heterogeneous between studies [15-19]. Therefore, further studies are required to identify which target genes/antigens detectable in peritoneal lavage fluid make the most optimal biomarkers for predicting outcome. Prospective validation of these biomarkers and validation studies to compare sensitivities and specificities between the various diagnostic criteria for positive cytology are also needed.

Conventional cytology is the most popular method because it is relatively inexpensive and requires neither the preservation of RNA nor the implementation of a complex technique. It has a high specificity, but often has a significantly lower sensitivity for detecting malignancy $[20,21]$. In this study, we used liquid-based cytology with ThinPrep processing, an automated cytopreparatory method. Our cytopathology laboratory has used the ThinPrep process as a standard cytology method for the preparation of all cytologic specimens since 2000. In conventional cytology, the large numbers of blood cells and various inflammatory cells in the peritoneal lavage fluid may obscure malignant cells. The ThinPrep technique can eliminate these disturbing factors and has been reported to show good correlation with conventional preparations and to reduce the rate of false-negative diagnoses [22, 23].

The objective of being able to detect intraperitoneal free malig- nant cells in patients with colorectal cancer, as well as gastric and ovarian cancer $[24,25]$, was to evaluate its impact on survival and recurrence and to discuss intraperitoneal treatment or adjuvant systemic chemotherapy. As a prognostic factor, positive malignant cells in peritoneal lavage fluid appear to be associated with poor overall survival and recurrence-free survival and with increased risk of peritoneal recurrence $[9,13]$. Although some studies have reported opposite results [10], majority of them had less than 100 patients and might be underpowered to show a difference in outcomes between positive and negative results [9].

To date, adjuvant chemotherapy has not been routinely recommended for low-risk stage-II patients. In the present study, four of the six patients with positive cytologic results were stage II. Although all of them received adjuvant chemotherapy, two patients showed recurrence: one at the peritoneum and the other at the peritoneum and the liver. If the strength of the association between positive peritoneal lavage and unfavorable outcome for recurrence and survival is considered, cytologic examination of peritoneal lavage fluid might be a useful tool to select the patients who need systemic or intraperitoneal chemotherapy and otherwise might not receive it. Noura et al. [8] reported in their analysis of 697 patients that positive peritoneal cytology was associated with poor prognosis and might be a useful marker for prediction of peritoneal recurrence. Furthermore, they showed that intraperitoneal chemotherapy with mitomycin $\mathrm{C}$ in positive cytology patients might reduce the peritoneal recurrence in their retrospective series [11]. In their study, postoperative intraperitoneal mitomycin $\mathrm{C}$ was given to 31 of the 52 patients with positive cytologic results who underwent curative surgery whereas adjuvant chemotherapy was 
administered to all patients with stages II and III cancer. After a mean follow-up of 83.1 months, the 31 patients who received intraperitoneal treatment had a significantly better peritoneal recurrence-free and cancer-specific survival rate compared with those who received systemic chemotherapy only [11]. No survival benefit with systemic chemotherapy has been reported in this situation. Further large prospective clinical trials are needed to investigate the role of intraperitoneal and systemic chemotherapy in patients with isolated positive cytology.

For other risk factors associated with peritoneal recurrence, there are ongoing similar clinical trials. One involves performing adjuvant HIPEC for patients with pathologic T4a cancer to reduce the risk of peritoneal recurrence [26]. The other involves performing a second-look laparotomy and HIPEC after a 1-year follow-up for patients with synchronous PC, ovarian metastasis or perforated colorectal cancer to detect early peritoneal recurrence [27]. However, in our opinion, there were some problems in performing those trials. The former trial had no accurate preoperative diagnostic tool to identify pathologic T4a tumors, and a pathologic examination to identify the T4a tumor had to be done within 1 day after surgery to perform the adjuvant HIPEC before starting postoperative adhesion. After development of postoperative adhesion in the dissection plane, the entrapment of any free intraperitoneal malignant cells might be possible, and those tumor cells would not be exposed to intraperitoneal chemotherapy drug. For the latter trial, although the effectiveness of cytoreductive surgery (CRS) and HIPEC is clear [12], the mortality and the morbidity of these aggressive therapies are relatively high. Reported postoperative mortality rates were $3.3 \%-3.8 \%$, and grades 3-4 complications occurred in $31 \%$ of the patients $[12,28]$. The expertise of the center had a strong impact on the prognosis because of the presence of a significant learning curve, so this is not a procedure that can be undertaken occasionally $[28,29]$. Considering these problems, we think that peritoneal lavage fluid cytology is a useful method to identify the cytologic result during surgery; therefore, intraperitoneal chemotherapy can be done immediately after resection of the tumor to reduce the peritoneal recurrence and further reduce the necessity for CRS and HIPEC, which have high mortality and morbidity. Because modern systemic chemotherapy with a biologic agent for PC has not shown improved outcomes compared with other site metastasis [4], prevention of peritoneal recurrence with early intraoperative chemotherapy might be a reasonable alternative treatment modality.

This study has some limitations of note. It is a single-institution observational study with a limited number of patients and a shortterm follow-up period. The major limitation of this study is that the number of patients with positive cytology was only six. Due to this small patient number, the $95 \%$ CIs of the HR for predicting the risks of death, overall recurrence, and peritoneal recurrence were so wide that the accuracies of the estimates of the HRs were deeply impaired, despite of the significant P-values. If the accuracies of the estimates of the HRs are to be raised, an increased the sample size is needed. Also, due to this small patient number, well- known risk factors of peritoneal recurrence, like T4 cancer or tumor perforation, did not show a significant association with positive cytology and peritoneal recurrence. Among the six patients with positive cytology, only 1 patient had pathologic T4 cancer, and only 1 patient had a tumor perforation. Thus, a type-II error might exist. In addition, the possibility of under-staging on the pathologic examination should be considered because controversy persists regarding the most appropriate criteria for diagnosing serosal invasion and because practical difficulties are associated with histological assessment in some cases [30].

In spite of the previously-described limitations of small number of patients with positive cytology, positive peritoneal lavage cytology was an independent predictive factor for peritoneal recurrence and an independent prognostic factor for poor DFS and OS. For further consideration of the role of peritoneal cytology, a large, population-based, multicenter study with long-term follow-up is required.

\section{CONFLICT OF INTEREST}

No potential conflict of interest relevant to this article was reported.

\section{REFERENCES}

1. Kanas GP, Taylor A, Primrose JN, Langeberg WJ, Kelsh MA, Mowat FS, et al. Survival after liver resection in metastatic colorectal cancer: review and meta-analysis of prognostic factors. Clin Epidemiol 2012;4:283-301.

2. Gonzalez M, Poncet A, Combescure C, Robert J, Ris HB, Gervaz P. Risk factors for survival after lung metastasectomy in colorectal cancer patients: a systematic review and meta-analysis. Ann Surg Oncol 2013;20:572-9.

3. Venook AP, Niedzwiecki D, Lenz HJ, Innocenti F, Mahoney MR, O'Neil BH, et al. CALGB/SWOG 80405: Phase III trial of irinotecan/5-FU/leucovorin (FOLFIRI) or oxaliplatin/5-FU/leucovorin (mFOLFOX6) with bevacizumab (BV) or cetuximab (CET) for patients with KRAS wild-type untreated metastatic adenocarcinoma of the colon or rectum [abstract]. In: 2014 American Society of Clinical Oncology Annual Meeting; 2014 May 30 - June 3; Chicago, USA. Alexandria (VA): American Society of Clinical Oncology; 2014.

4. Kerscher AG, Chua TC, Gasser M, Maeder U, Kunzmann V, Isbert $\mathrm{C}$, et al. Impact of peritoneal carcinomatosis in the disease history of colorectal cancer management: a longitudinal experience of 2406 patients over two decades. Br J Cancer 2013;108:1432-9.

5. Koppe MJ, Boerman OC, Oyen WJ, Bleichrodt RP. Peritoneal carcinomatosis of colorectal origin: incidence and current treatment strategies. Ann Surg 2006;243:212-22.

6. Keettel WC, Elkins HG. Experience with radioactive colloidal gold in the treatment of ovarian carcinoma. Am J Obstet Gynecol 1956; 71:553-68. 
7. Edge SB, Byrd DR, Compton CC, Fritz AG, Greene FL, Trotti A, editors. AJCC cancer staging manual. 7th ed. New York: Springer; 2010.

8. Noura S, Ohue M, Seki Y, Yano M, Ishikawa O, Kameyama M. Longterm prognostic value of conventional peritoneal lavage cytology in patients undergoing curative colorectal cancer resection. Dis Colon Rectum 2009;52:1312-20.

9. Mohan HM, O'Connor DB, O'Riordan JM, Winter DC. Prognostic significance of detection of microscopic peritoneal disease in colorectal cancer: a systematic review. Surg Oncol 2013;22:e1-6.

10. Cotte E, Peyrat P, Piaton E, Chapuis F, Rivoire M, Glehen O, et al. Lack of prognostic significance of conventional peritoneal cytology in colorectal and gastric cancers: results of EVOCAPE 2 multicentre prospective study. Eur J Surg Oncol 2013;39:707-14.

11. Noura S, Ohue M, Shingai T, Kano S, Ohigashi H, Yano M, et al. Effects of intraperitoneal chemotherapy with mitomycin $\mathrm{C}$ on the prevention of peritoneal recurrence in colorectal cancer patients with positive peritoneal lavage cytology findings. Ann Surg Oncol 2011;18:396-404.

12. Verwaal VJ, Bruin S, Boot H, van Slooten G, van Tinteren H. 8-year follow-up of randomized trial: cytoreduction and hyperthermic intraperitoneal chemotherapy versus systemic chemotherapy in patients with peritoneal carcinomatosis of colorectal cancer. Ann Surg Oncol 2008;15:2426-32.

13. Bosanquet DC, Harris DA, Evans MD, Beynon J. Systematic review and meta-analysis of intraoperative peritoneal lavage for colorectal cancer staging. Br J Surg 2013;100:853-62.

14. Altomare DF, Tedeschi M, Rotelli MT, Bocale D, Piscitelli D, Rinaldi $\mathrm{M}$. Lack of prognostic role of pre- and postoperative peritoneal cytology and cytokeratin PCR-expression on local recurrence after curative anterior resection for mid-low rectal cancer. Updates Surg 2011;63:109-13.

15. Kowalewska M, Chechlinska M, Nowak R. Carcinoembryonic antigen and cytokeratin 20 in peritoneal cells of cancer patients: are we aware of what we are detecting by mRNA examination? Br J Cancer 2008;98:512-3.

16. Kristensen AT, Wiig JN, Larsen SG, Giercksky KE, Ekstrom PO. Molecular detection (k-ras) of exfoliated tumour cells in the pelvis is a prognostic factor after resection of rectal cancer? BMC Cancer 2008;8:213.

17. Lloyd JM, McIver CM, Stephenson SA, Hewett PJ, Rieger N, Hardingham JE. Identification of early-stage colorectal cancer patients at risk of relapse post-resection by immunobead reverse transcriptionPCR analysis of peritoneal lavage fluid for malignant cells. Clin Cancer Res 2006;12:417-23.

18. Bosch B, Guller U, Schnider A, Maurer R, Harder F, Metzger U, et al. Perioperative detection of disseminated tumour cells is an independent prognostic factor in patients with colorectal cancer. $\mathrm{Br} \mathrm{J}$
Surg 2003;90:882-8.

19. Vogel I, Francksen H, Soeth E, Henne-Bruns D, Kremer B, Juhl H. The carcinoembryonic antigen and its prognostic impact on immunocytologically detected intraperitoneal colorectal cancer cells. Am J Surg 2001;181:188-93.

20. Stewart CJ, Mills PR, Carter R, O’Donohue J, Fullarton G, Imrie $\mathrm{CW}$, et al. Brush cytology in the assessment of pancreatico-biliary strictures: a review of 406 cases. J Clin Pathol 2001;54:449-55.

21. Volmar KE, Vollmer RT, Routbort MJ, Creager AJ. Pancreatic and bile duct brushing cytology in 1000 cases: review of findings and comparison of preparation methods. Cancer 2006;108:231-8.

22. Abulafia O, Pezzullo JC, Sherer DM. Performance of ThinPrep liquid-based cervical cytology in comparison with conventionally prepared Papanicolaou smears: a quantitative survey. Gynecol Oncol 2003;90:137-44.

23. Clayton AC, Bentz JS, Wasserman PG, Schwartz MR, Souers RJ, Chmara BA, et al. Comparison of ThinPrep preparations to other preparation types in gastrointestinal cytology: observations from the College of American Pathologists Interlaboratory Comparison Program in Nongynecologic Cytology. Arch Pathol Lab Med 2010; 134:1116-20.

24. Cabalag CS, Chan ST, Kaneko Y, Duong CP. A systematic review and meta-analysis of gastric cancer treatment in patients with positive peritoneal cytology. Gastric cancer 2014 Jun 3 [Epub]. Available from: http://dx.doi.org/10.1007/s10120-014-0388-5.

25. Simojoki M, Santala M, Vuopala S, Kauppila A. The prognostic value of peritoneal cytology in ovarian cancer. Eur J Gynaecol Oncol 1999;20:357-60.

26. Hompes D, Tiek J, Wolthuis A, Fieuws S, Penninckx F, Van Cutsem E, et al. HIPEC in T4a colon cancer: a defendable treatment to improve oncologic outcome? Ann Oncol 2012;23:3123-9.

27. Ripley RT, Davis JL, Kemp CD, Steinberg SM, Toomey MA, Avital I. Prospective randomized trial evaluating mandatory second look surgery with HIPEC and CRS vs. standard of care in patients at high risk of developing colorectal peritoneal metastases. Trials 2010;11:62.

28. Elias D, Gilly F, Boutitie F, Quenet F, Bereder JM, Mansvelt B, et al. Peritoneal colorectal carcinomatosis treated with surgery and perioperative intraperitoneal chemotherapy: retrospective analysis of 523 patients from a multicentric French study. J Clin Oncol 2010;28:63-8.

29. Smeenk RM, Verwaal VJ, Zoetmulder FA. Learning curve of combined modality treatment in peritoneal surface disease. Br J Surg 2007;94:1408-14.

30. Stewart CJ, Hillery S, Platell C, Puppa G. Assessment of serosal invasion and criteria for the classification of pathological (p) T4 staging in colorectal carcinoma: confusions, controversies and criticisms. Cancers (Basel) 2011;3:164-81. 